\title{
Fin de vie: tension entre principes théoriques et réalité clinique
}

\section{Jean Martin}

Dr méd., membre de la rédaction

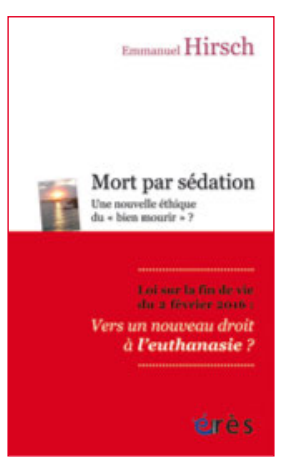

Emmanuel Hirsch

Mort par sédation Une nouvelle éthique du «bien mourir»? Toulouse: Editions érès; 2016.

209 pages. $19.70 \mathrm{CHF}$. ISBN 978-2-749-25223-0

jean.martin[at]saez.ch
Emmanuel Hirsch est un intervenant marquant en bioéthique francophone depuis trente ans. Il a écrit ou dirigé de nombreux ouvrages et dirige l'Espace de réflexion éthique de la région Ile-de-France. Son dernier livre est une publication qu'on peut dire militante, quelque peu amère dans la mesure où il y présente en détail ses réserves, en fait son opposition, aux modifications apportées le 2 février 2016 à la loi Leonetti de 2005 (devenue ainsi loi Claeys-Leonetti) créant de nouveaux droits en faveur des personnes en fin de vie. Sa crainte est que, avec la sédation terminale ainsi incluse au cadre légal, on s'achemine vers l'acceptation de l'euthanasie, comme au Benelux, ou vers le suicide médicalement assisté, comme en Suisse (auxquels certains semblent prêts à retirer la qualité de pays civilisés).

L'auteur a été très impliqué dans les débats qui ont précédé l'adoption de dite loi. Et il regrette vivement que ceux qui avec lui ont les mêmes réserves n'aient pas été assez entendus par les instances concernées puis par le parlement français. Cela étant, on souhaiterait que ceux qui critiquent les modifications admises, et l'évolution dont elles font partie, se penchent plus avant sur la réalité au lit du malade. Ainsi sur le caractère insuffisamment pertinent, inapproprié même (notamment en soins intensifs), de distinctions sur lesquelles on a beaucoup insisté au plan théorique. La formule «Laisser mourir, oui, faire mourir jamais» est théoriquement parfaite mais, de plus en plus souvent en pratique, n'est pas un guide adéquat, tant la limite entre l'un et l'autre devient floue - une insistance dogmatique sur ce point peut certainement aller à l'encontre de l'accompagnement le plus approprié. Emmanuel Hirsch en est d'ailleurs conscient quand il relève que «les techniques de réanimation ont rendu parfois indistincte la frontière entre vie et survie artificielle». En fait, ce n'est pas "parfois», c'est la réalité fréquente d'une médecine qui dans des situations irréversibles peut maintenir indéfiniment l'existence. Mettre l'accent sur le caractère déterminant de l'intentionnalité d'une mesure n'est pas plus aidant et risque en fait de ne servir qu'à stigmatiser (alors que la notion du «double effet» potentiel est admise par tous, y compris l'Eglise catholique). Hirsch questionne aussi la conclusion "On meurt mal en France» du Rapport de la commission Sicard de décembre 2012, souvent cité. Pourtant, dite conclusion est largement admise - même s'il y a sans doute aussi des institutions exemplaires. Un autre point majeur est la notion d'abandon: pour l'auteur, c'est abandonner le patient que de permettre la sédation terminale; dans la pratique, on rencontre aussi des malades qui jugent qu'on les abandonne en les laissant souffrir jusqu'à la dernière extrémité - et qui serions-nous pour les disqualifier?

Par moments, on se demande pourquoi l'auteur a tenu à écrire cet ouvrage sur une lutte qu'il dit lui-même perdue. "Pour que demeurent les traces d'un engagement dont je constate aujourd'hui l'inanité», dit-il. Complètement respectable bien sûr, mais, au vu de ses contributions antérieures dans le domaine, une autre modalité aurait été de participer, de manière critique mais constructive, à la définition de ce qui vaudra demain. En $4^{\mathrm{e}}$ page de couverture est posée la

\section{«Pour que demeurent les traces d'un engage- ment dont je constate aujourd'hui l'inanité.»}

question: «N'aurait-il pas été alors plus sage et courageux de créer les conditions effectives d'un choix possible entre un accompagnement humain jusqu'au terme de la vie et une euthanasie par compassion?». Il est vraisemblable que l'avenir est dans ce sens. A ceci près qu'il n'y a pas lieu de choisir l'un ou l'autre mais qu'il est tout à fait possible d'avoir un accompagnement digne, d'une part, et d'autre part, quand les circonstances précisément le rendent humain et compréhensible, d'ouvrir la possibilité d'une assistance médicale au suicide - voire une euthanasie. Sur la demande instante et répétée du patient capable de discernement. A cet égard, et en milieu universitaire francophone, on peut faire référence aux travaux du professeur de soins palliatifs G. D. Borasio, de Lausanne. 